\title{
What is the Best Achievable QoS for Unicast Routing in VANET?
}

\author{
Mate Boban ${ }^{1,2}$, Geoff Misek ${ }^{1}$, and Ozan K. Tonguz ${ }^{1}$ \\ ${ }^{1}$ Department of Electrical and Computer Engineering \\ Carnegie Mellon University \\ Pittsburgh, PA, 15213-3890, USA \\ ${ }^{2}$ Faculty of Organization and Informatics \\ University of Zagreb \\ Varazdin, Croatia \\ mboban@cmu.edu,gmisek@cmu.edu, tonguz@ece.cmu.edu
}

\begin{abstract}
Significant efforts and studies were recently reported for enabling active safety, traffic management, and commercial applications in Vehicular Ad Hoc Networks (VANET), since these applications are the drivers of the recent surge in VANET research and development. However, very few research efforts considered analyzing the Quality of Service (QoS) metrics that will be available to these applications in VANET. Furthermore, although there are many proposed solutions for routing in VANET, it is still unclear as to what specific characteristics VANET routing protocols should possess, since none of the proposed solutions achieves optimum performance in both urban and highway, as well as sparse and dense environment. To shed light on these issues, in this paper we analyze some of the most important QoS metrics in VANET. Namely, we determine the upper performance bound for connection duration, packet delivery ratio, end-to-end delay, and jitter for unicast communication in typical highway and urban VANET environments. According to our results, delay and jitter in VANET would be adequate for most of the envisioned unicast-based applications, whereas the packet delivery ratio and connection duration might not meet the requirements for most unicast-based applications.

Index Terms-VANET, vehicular ad hoc networks, QoS, connectivity, realistic mobility model, simulation, inter-vehicle communication, real-time applications, routing.
\end{abstract}

\section{INTRODUCTION}

Vehicle to vehicle (V2V) communication is possible today with Vehicular Ad hoc NETworks (VANET). This capability has so far been envisioned for safety and traffic management reasons: vehicles can inform other vehicles of hazardous road conditions, traffic congestion, or sudden stops. In order to create incentive for faster adoption of the technology, commercial services (e.g., infotainment, audio/video streaming, V2V multiplayer gaming, etc.) are also envisioned in VANET [1].

Although a certain degree of infrastructure support for VANET (e.g., Roadside Units, sensors, cameras, etc.) is envisioned, thus making vehicle-to-infrastructure (V2I) communication possible, at this point it is obvious that in the initial phase of VANET deployment vehicles will often not be able to rely on the infrastructure support. One of the arguments corroborating this claim is the U.S. DOT infrastructure deployment plan [2]. Therefore, in this paper we take the viewpoint that $\mathrm{V} 2 \mathrm{~V}$ communication will play a crucial role in VANET, often being the only possible communication paradigm. This is especially true in highway environments, where full infrastructure support would incur very high cost to deploy and maintain. We therefore assume no infrastructure support in this paper.

Cheng and Robertazzi showed in [3] that the probability of end-to-end connectivity in infrastructureless wireless networks, such as VANET, decreases with distance as a function of node's transmission range and node density. We therefore believe that applications in VANET will exhibit locality of interest, where vehicles will only communicate with other vehicles that are relatively close to them (i.e., reachable via a reasonable number of hops). Relaying messages over a large area without infrastructure support (i.e., over a large number of intermediate vehicles) would have a negative effect on the delay and delivery ratio of messages. These facts imply that applications in infrastructureless VANET, particularly the ones not dealing with safety, will be bound to a certain region of interest in which the communication will be feasible.

Dedicated Short Range Communications (DSRC), specified under IEEE standard $802.11 \mathrm{p}$, is becoming the de facto standard for physical (PHY) and medium access control (MAC) layers of the VANET proposed communication stack, Wireless Access in Vehicular Environment (WAVE), defined in IEEE 1609.x family of standards. Atop the DSRC, we implemented a routing solution that enables us to analyze the potential performance of unicast routing as well as Quality of Service (QoS) metrics available to applications requiring unicast communication. Based on a simulation environment with realistic mobility model and wireless signal propagation and by implementing the optimized routing solution over the PHY and MAC layers of the DSRC trial standards, we analyzed the following QoS parameters: connection duration, packet delivery ratio (PDR), end-to-end delay, and jitter. Simulations were performed in 
both highway and urban environments with different traffic densities and relative speeds in order to encompass a wide variety of urban and highway scenarios.

Because of the specific metrics analyzed, and given the fact that we are exclusively interested in analyzing unicast message exchange (i.e., specific sender and receiver of a message instead of message broadcast), the results we present primarily pertain to the feasibility of unicast-based real-time applications - ones that require the delay, jitter, PDR, and connection duration to be within specific boundaries for the application to be feasible.

Our main contributions are as follows:

- QoS Analysis. Based on the large scale simulations, employed routing solution, and by implementing DSRC at PHY and MAC layers, we are able to perform detailed analysis of the QoS parameters as well as the attainable unicast-based application performance over infrastructureless VANET in both highway and urban environments.

- Connectivity Analysis. Besides the 'usual' QoS constraints, applications in VANET have to take into the consideration the scarcity of connectivity in such a highly dynamic network. To that end, we analyze the connection duration for typical scenarios in both highway and urban environments.

- VANET Routing Protocol Dynamics. By implementing the routing that allows the analysis of all the available routes and incurs no unnecessary delay and overhead, we determine the upper bound of the performance that any unicast routing protocol, and thus any applications running on top of it, can achieve over DSRC-enabled VANET.

The rest of the paper is organized as follows. Section II gives a brief overview of the related work and elaborates on our contribution. Section III discusses in detail the functioning of the implemented routing solution. The simulation setup, different highway and urban scenarios, and studied performance metrics are presented in Section IV. Obtained results and the discussion are given in Section V. Section VI points out the future work and concludes the paper.

\section{RELATED WORK}

With regards to the QoS metrics for real-time applications in VANET, Wang in [4] discussed the connection duration, route lifetime, and route repair frequency in VANET. The simulations were conducted on a closed rectangular highway with an unrealistically small transmission range (from 100 to 150 $m$ ) for highway environment. Properly defining transmission range is important because it was shown to affect the connectivity in an exponential fashion [5]. Furthermore, there is no indication of which PHY and MAC protocols were used for the simulation. Bai et al. in [6] presented simulation results based on several mobility models and different routing protocols. They characterized route duration and the effect of mobility on routing protocol performance. The mobility models used included the highway and urban environment represented by a perfect grid. Artimy et al. in [7] analyzed connectivity by simulating the node movement using a cellular automata model, while Rudack et al. in [8] presented a simple analytical framework for single-hop connection duration in VANET and provided experimental evaluation of the framework. Ho et al. in [9] analyzed structured mobility in urban environment and determined the impact of multihop communication and density on connectivity and path stability. Mabiala et al. in [10] analyzed connection duration on highways. The simulations were run over existing routing protocols, and with a simplified simulation setup. In a recent study, Fiore and Härri in [11] analyzed the link (i.e., one hop) duration as a function of different mobility models and road layouts. With respect to determining the feasibility of different applications in VANET, Yin et al. in [12] presented a comprehensive feasibility study of delay-critical safety applications over VANET by analyzing the physical characteristics of DSRC. Namely, the analyzed metrics encompass bit error rate, throughput, and delay. Feasibility studies for various non-safety applications were conducted in [13], [14], and [15].

In this paper we are interested in describing the routing dynamics in VANET, taking into account the proposed underlying DSRC layers. Naumov et al. in [16] presented realistically modeled simulations based on the microscopic traffic simulator and ns-2 network simulator. The authors employed novel enhancements to reactive routing protocols and analyzed delay, PDR, and network load. Several other research efforts focused on providing higher level of QoS in VANET by implementing more efficient routing protocols (e.g., [17] and [18]).

All of the efforts above pertain to specific types of routing protocols, whereas in this paper we take a different approach: we are interested in defining the upper boundary that any unicast routing protocol can strive for in infrastructureless, DSRC-enabled VANET. By abstracting the routing protocol in such a way, we are able to obtain valuable information regarding the achievable performance for routing protocols in VANET. Additionally, to the best of our knowledge, a comprehensive analysis of the QoS metrics for unicast-based applications in the infrastructureless VANET environment with realistic simulation parameters (based on DSRC, actual roads, and credible mobility patterns), and realistic signal propagation modeling has not been conducted.

\section{EMPloyed ROUTING SOLUTION}

Substantial research efforts are being undertaken to create VANET routing protocols that will exhibit a high level of performance in both urban and highway environments, as well as in dense and sparse vehicular networks [19]. Despite that, at this point it is still not clear which characteristics routing protocols should possess in order to perform adequately in a diverse and fast changing VANET environment. The motivation behind this paper is to determine the optimum performance in infrastructureless VANET achievable by any 
unicast routing protocol over DSRC, since DSRC is the de facto standard for PHY and MAC layers in VANET.

We employed a routing solution that assures message delivery if the underlying DSRC layers relay the message successfully. The solution is based on a scheme where a node (vehicle) relays the message if it has not relayed the same message before. Changing routes usually implies additional overhead for all state-based routing protocols, and more often than not there are lost messages in the process of route repair (from the time the route is broken until the next route is found). The implemented solution is able to choose between all available routes instantaneously, which implies that there is no additional delay or packet loss during the route change; this corresponds to an ideal case where the routing protocol switches routes seamlessly.

In a typical VANET scenario, numerous concurrent communication sessions will take place between different sender/receiver pairs for unicast applications. Clearly, the interplay between sessions will introduce additional delay and jitter, and will potentially result in loss of packets. In order to analyze the best achievable unicast performance over VANET, in this paper we consider only one sender/receiver pair per simulation run; this enables us to determine the minimum delay and jitter, since there are no other packets in the network aside from the ones generated by the observed sender/receiver pair. Furthermore, we do not observe packet collisions in any of our simulation runs. Thus, we can conclude that the packet loss occurs solely because of the nonexistence of a physical path between the sender and the destination, and the communication is uninterrupted while a path between the sender and receiver exists.

To illustrate the principle of operation of the employed solution, Fig. 1 presents the following situation. Suppose that at time $t_{1} P a t h \# 1$ is the optimum path (i.e., delivers the message $m_{1}$ to the destination with the least amount of delay). If at time $t_{2}$, due to the fluctuating environment, Path\#2 becomes the optimum path (i.e., delivers the message $m_{2}$ with the least amount of delay), the employed scheme will switch between these paths seamlessly (without additional delay or loss of messages). Next, suppose that at time $t_{3}$ the only available path is Path\#3. If at time $t_{4}$, the direct link between the sender $S$ and the relay node $R_{1}$ becomes unavailable and the links $\left[S-R_{2}\right],\left[R_{2}-R_{3}\right]$, and $\left[R_{3}-R_{1}\right]$ are available, the message $m_{4}$ will be delivered over the newly created path (Path\#3') without the loss or additional delay. In this manner, the solution ensures message delivery through the optimum path at any point in time. As noted earlier, no packet loss due to collision was observed in our simulation which implies that, when there exists a physical path between the sender and receiver, a) there is no difference between the employed routing scheme and the ideal routing ${ }^{1}$ with respect to the PDR and the connection duration, and $\mathrm{b}$ ) the only difference to the ideal routing with respect to the delay and jitter is due to the

\footnotetext{
${ }^{1}$ Ideal routing is interpreted as the perfect routing which incurs no additional loss, delay, or overhead.
}

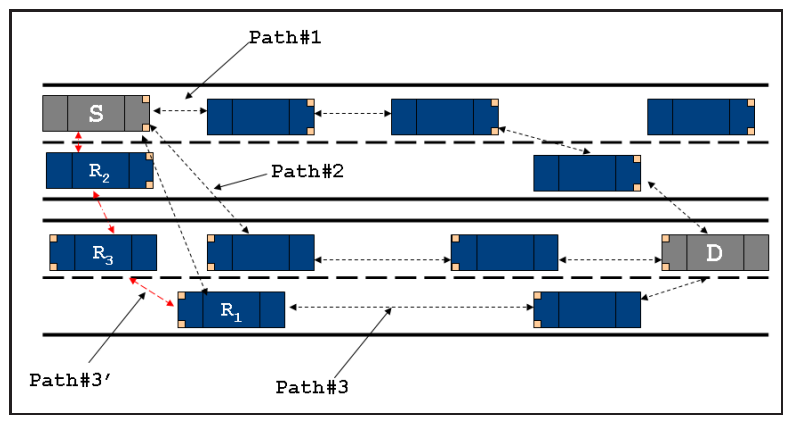

Fig. 1. Seamless switching between optimum paths.

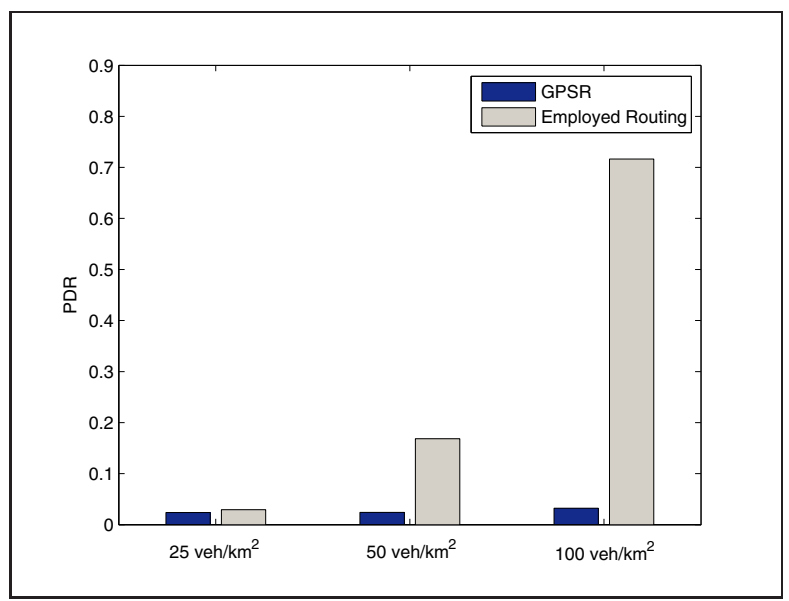

Fig. 2. PDR of GPSR and employed routing in urban environment.

practically negligible channel contention which is measured in microseconds.

To shed some light on the impact of a routing protocol on performance in VANET, we ran simulations with both our solution and the GPSR routing protocol with omniscient location service [20]. The simulation setup was identical in both cases: DSRC underlying the routing protocol with vehicles moving over the identical urban area. Fig. 2 shows performance differences between the employed protocols, ranging from 23 percent less messages delivered using the GPSR in low vehicle density scenario ( $25 \mathrm{veh} / \mathrm{km}^{2}$ ), up to 20 times less in higher vehicle density scenario (100 veh/ $\mathrm{km}^{2}$ ). With GPSR, we observed significantly increased use of perimeter mode as the vehicle density increased. This implies that the increase in PDR discrepancy as the vehicle density increases is due to the GPSR's inability to determine the optimum next hop as the number of available neighboring vehicles becomes larger, thus often resorting to perimeter mode which eventually results in frequent packet drops. At a low vehicle density $\left(25 \mathrm{veh} / \mathrm{km}^{2}\right)$, GPSR does not suffer from this issue since there are usually only a few neighboring nodes and next hop selection is close to optimal.

\section{Simulation Setur}

Given the high cost of deploying actual equipment in the vehicles, real life testbeds for VANET research are extremely 
rare; this is especially true for medium and large scale systems, which are practically nonexistent, thus making the simulation environments the only feasible tools for analyzing the fundamental properties of VANET. We conducted simulations using the Jist/SWANS simulator with the STRAW mobility model. JiST (Java in Simulation Time) is a discrete event simulation environment, and SWANS (Scalable Wireless Ad Hoc Network Simulator) is a publicly available Java-based scalable wireless network simulator [21]. STRAW (STreet RAndom Waypoint) [22] is a vehicular mobility model, built on top of the JiST/SWANS platform, that constrains the node movement to real U.S. streets (based on the U.S. Census Bureau's TIGER data [23]). STRAW implements the carfollowing model [24] with lane changing, intersection control, and supports flows of vehicles (groups of vehicles with the same starting and destination point).

\section{A. Simulation parameters}

As noted earlier, we implemented the DSRC PHY and MAC layers based on the IEEE 802.11p draft standard [25], and we used connectionless unacknowledged (Type 1) operation of the LLC as specified in the IEEE standard 802.2. We do not make use of DSRC's multi-channel functionality, since we only analyze a single sender/receiver pair. Atop the DSRC, we implement the routing scheme described in Section III. We do not make any assumptions regarding the transport protocol; this is another ongoing VANET research topic which is beyond the scope of this paper. Therefore, in the simulation we encapsulate the application-layer data directly in IP datagrams.

In order to set up a testbed as credible as possible, we followed the simulation modeling recommendations and tried to avoid the erroneous assumptions described by Kotz et al. in [26]: a radio's transmission area is circular; all radios have equal range; if I can hear you, you can hear me (symmetry); if I can hear you at all, I can hear you perfectly; signal strength is a simple function of distance. Detailed simulation parameters are presented in Table $\mathrm{I}^{2}$.

For signal propagation in the highway environment, we used the two-ray path loss model with a transmission range of $550 \mathrm{~m}$. The transmission range was based on the the recent field testing of DSRC equipment reported in [27], while [28] indicated that the signal propagation on highways can be adequately modeled with a deterministic two-ray path loss. In the urban environment, on the other hand, we implemented the probabilistic shadowing model, which better describes the complex urban surroundings with numerous obstacles (buildings, trees, overpasses, etc.). Using a stochastic approach to calculate the receiving power, and by taking into account the multipath propagation effects, shadowing model can describe the urban environment more realistically. The shadowing model is described by $\left[\frac{P_{w}(d)}{P_{w}\left(d_{0}\right)}\right]_{d B}=-10 \beta \log \left(\frac{d}{d_{0}}\right)+X_{d B}$, where $\beta$ is the path loss exponent, $X_{d B}$ is a normally

\footnotetext{
${ }^{2} \mathrm{~A}$ density of zero $v e h / \mathrm{km}$ indicates that there were no vehicles present in the scenario, except for the two observed vehicles.
}

distributed random variable with mean zero and deviation $\sigma_{d B}, P_{w}(d)$ is the power at the receiver, and $P_{w}\left(d_{0}\right)$ is the reference power received at a close distance $d_{0}$. By varying the values of $\beta$ and $\sigma_{d B}$, it is possible to simulate environments with different characteristics (from a flat world, free space signal propagation, to heavily shadowed environment with large number of obstacles and significant multipath effects).

Since we were interested in modeling the typical unicast real-time message exchange scenarios, the message size and frequency were chosen based on the measurements conducted in [29]. The simulation time for all scenarios was 600 seconds, with an additional warm-up time of 200 seconds during which no packets were sent, in order to have a more realistic initial vehicle distribution. We executed simulations with a total of more than 240 simulation hours. All of the nodes in the simulations were equipped with DSRC radios, and DSRC parameters were set up based on [25] and [27]. We opted not to perform simulations with varying penetration ratio because the recent connectivity study based on the traffic data collected from interstate I-80 in California showed that decreasing the penetration ratio is equivalent to decreasing the traffic volume, which leads to the conclusion that, at a certain market penetration ratio below $100 \%$, the network connectivity will be the same as with $100 \%$ market penetration, given the equivalent increase in traffic volume [17]. To that end, we employ relatively low vehicle densities in our simulations in order to account for the lower penetration ratios that will occur during the initial phases of VANET deployment.

\section{B. Highway and urban environment}

We ran our experiments under two broad categories: highway environment and urban environment. Based on the concept of locality of interest, we set up our simulations in order to determine the QoS metrics between vehicles that are within each others' multihop communication range as opposed to randomly selecting senders and receivers, which could be so far away from each other that even an unrealistically large 50 hop route would not be able to connect them.

1) Highway: For the highway environment, we ran the simulations on a segment of I-80 in Elko County, NV, presented in Fig. 3a, with equivalent simulation visualization in Fig. 3b (vehicle icons are not drawn to scale). The roadway portion we used was approximately $43.5 \mathrm{~km}$ long, with 3 lanes per direction. Within the simulation, we chose two vehicles as the observed vehicles (vehicles selected to be the sender/receiver pair in a given simulation run). For these vehicles, we specified initial placement, speed, and travel direction. We distinguished same direction and opposite direction scenarios. In the same direction scenarios, the observed vehicles were initially placed next to each other and moved in the same direction. In the opposite direction scenarios, the observed vehicles were placed approximately $10 \mathrm{~km}$ apart and moved towards each other. To have a greater control over the simulation, we specified the relative speed (difference between the speeds) of the two observed vehicles as $0,5,10$, and $15 \mathrm{~m} / \mathrm{s}$ in the same direction 
TABLE I

SIMULATION PARAMETERS

a) Global parameters

\begin{tabular}{|c|c|}
\hline Parameter & Value \\
\hline Number of senders & 2 (sending messages to each other) \\
\hline Number of messages & 20 per second \\
\hline Message size & $\begin{array}{c}\text { Normally distributed } \\
\text { mean }=100 \mathrm{~B} ; \text { variance }=15 \mathrm{~B}\end{array}$ \\
\hline TTL (max. \# of hops allowed) & 20 \\
\hline Vehicle densities (highway) & $0,1,5,10 \mathrm{veh} / \mathrm{km}$ \\
\hline Vehicle densities (urban) & $\begin{array}{c}10,25,50,100 \mathrm{veh} / \mathrm{km}^{2} \\
\text { Normally distributed } \\
\text { Speeds of vehicles } \\
\text { variance (highway) }=25 \% \text { mean } \\
\text { variance (urban) }=35 \% \text { mean }\end{array}$ \\
\hline Simulation duration & 600 seconds \\
\hline
\end{tabular}

\section{b) DSRC parameters}

\begin{tabular}{|c|c|}
\hline Parameter & Value \\
\hline Frequency & $5.9 \mathrm{GHz}$ \\
\hline Channel size & $20 \mathrm{M} \mathrm{Hz}$ \\
\hline Transmission rate & $6 \mathrm{Mb} / \mathrm{s}$ \\
\hline Transmission power & $20 \mathrm{dBm}$ \\
\hline Radio reception threshold & $-84.7 \mathrm{dBm}$ \\
\hline
\end{tabular}

c) Signal propagation parameters

\begin{tabular}{|c|c|}
\hline Parameter & Value \\
\hline Signal propagation model (highway) & Two-ray \\
\hline Transmission range (highway) & $550 \mathrm{~m}$ \\
\hline Signal propagation model (urban) & Shadowing \\
\hline Shadowing parameters & $\beta=2.6 ; \sigma_{d B}=6$ \\
\hline
\end{tabular}

and as $40,50,60$, and $70 \mathrm{~m} / \mathrm{s}$ in the opposite direction scenarios. These values encompass the usual speed deviation on the U.S. highways [30]. It is important to note that although the observed vehicles attempted to move at the speeds noted above, they obeyed the employed mobility model [22]; thus, their speeds changed according to the traffic conditions, and the relative speed values noted above are to be considered as a rough guidance while analyzing the obtained data.

The remaining non-observed vehicles were placed on the highway randomly based on a uniform distribution, and they traveled in a randomly chosen direction. Their speeds were set based on a normal distribution with the mean speed equal to the actual speed limit of the I-80 in Elko county (approx. 30 $\mathrm{m} / \mathrm{s}$ ), and a variance of $25 \%$ of the mean speed. The variance was determined with regards to the empirical measurements of vehicles' speed and variance on highways reported in [30]. The number of vehicles in the highway simulations was chosen to create the global densities specified in Table Ia.

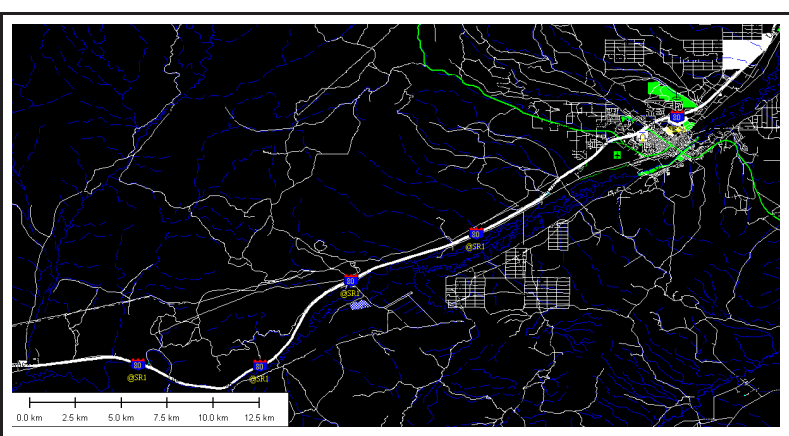

(a) TIGER map

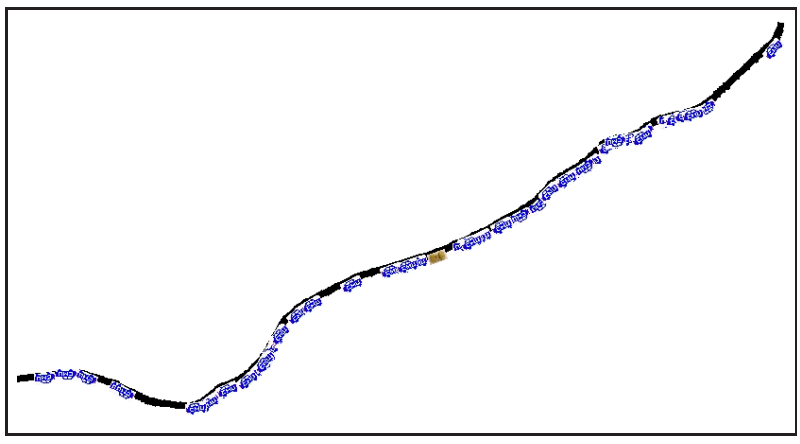

(b) Simulation visualization

Fig. 3. Highway area - Elko County, NV.

2) Urban: For the urban environment, we used a portion of the city area of Chicago presented in Fig. 4a, with equivalent simulation visualization in Fig. 4b (again, vehicle icons are not drawn to scale). This area was approximately $2.3 * 3.2 \mathrm{~km}^{2}$. We analyzed scenarios with different vehicle densities per square kilometer, as specified in Table Ia. Similar to the highway environment, we chose two vehicles as the observed vehicles. In urban environment, both observed and non-observed vehicles were placed randomly based on the uniform distribution and traveled on a random route according to the STRAW mobility model. The speeds of both observed and non-observed nodes were randomly chosen from a normal distribution with the mean speed equal to the actual speed limit of the current road the vehicle is traversing and the variance of the speed set to $35 \%$ of the mean speed in order to mimic a more volatile urban environment. The mean speed varied, since there were different road types in the simulated area.

\section{Studied performance metrics}

1) Connection duration: In order to have a meaningful interaction between different parties, most applications require a connection duration to last a certain amount of time. For instance, a live audio or video streaming session can last a variable amount of time, and during that time the connection between the publisher and subscriber must be available. Similarly, a multiplayer game session can last anywhere from one minute up to several hours depending on the type of the game.

In the same direction highway scenarios we report double the observed value of connection duration for all relative 


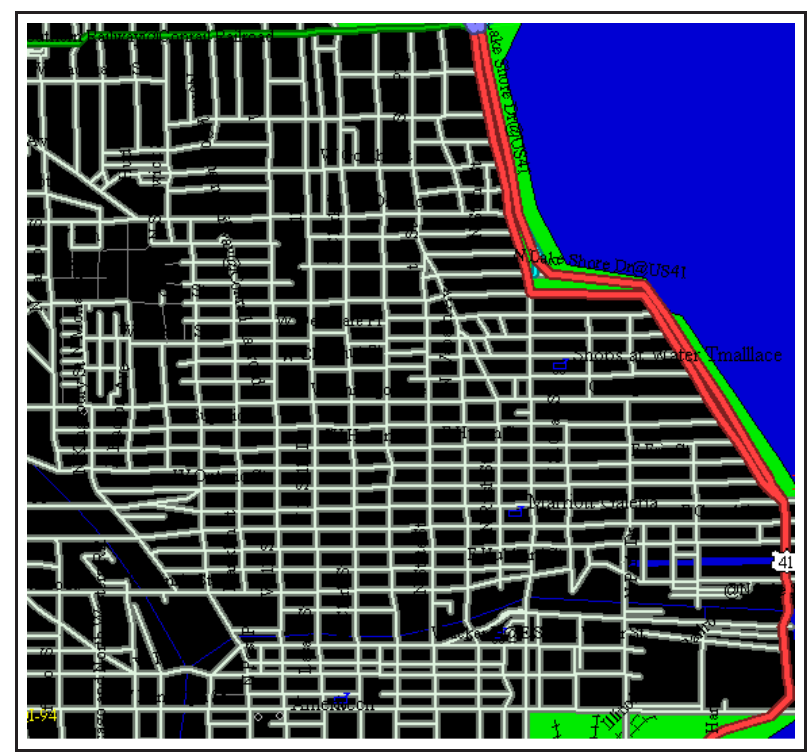

(a) TIGER map

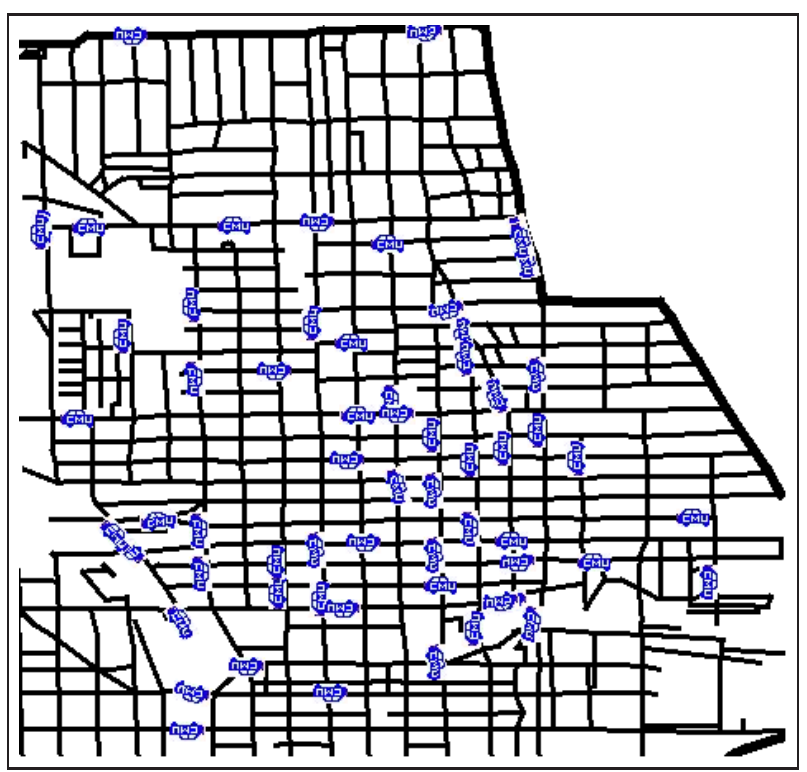

(b) Simulation visualization

Fig. 4. Urban area - Chicago.

speeds except the relative speed zero. The reasoning behind this is as follows. The observed nodes travel in the same direction with the specified speeds. Furthermore, the expected number of the relaying non-observed nodes is equal before and after the observed nodes meet thus implying that, on average, there will be the same number of nodes to relay the messages before and after the observed nodes meet. That is why, on average, we expect a symmetric connection duration distribution before and after the observed nodes meet. However, note that we initially place the vehicles next to each other, thus only measuring one half of the symmetric connection duration. Therefore, the observed value of connection duration will correspond to one half of the actual connection duration.
2) End-to-end delay: Many applications have strict constraints on maximum tolerable delay. For instance, various time-critical VANET safety applications, such as intersection collision warning, require end-to-end delay to be under 100 $m s$ [31]. Similarly, non-safety applications such as fast paced action multiplayer games [29] can only tolerate up to 150 $m s$ of end-to-end delay, while applications such as streaming audio/video and turn-based multiplayer games tolerate up to several seconds of delay. In this paper, we report the one way end-to-end delay.

3) Jitter: Jitter is the variation in the end-to-end delay between packets arriving at the destination. In real-time applications such as VoIP, video conferencing, and multiplayer games, jitter is often considered as important of a metric as end-to-end delay [29].

4) Packet delivery ratio: Packet delivery ratio is the ratio of the number of messages received by the destination to the number sent by the sender. Since we believe that applications in infrastructureless VANET (especially real-time applications) will display locality of interest, we only analyze the packet delivery ratio for the urban scenario; the highway scenario is simulated over 43.5 kilometers of roadway, and assuming that two nodes 43.5 kilometers apart would be able to communicate without the infrastructure is not realistic, since a message relayed over such a large area would incur high delay and network load.

\section{Simulation Results and Discussion}

Fig. 5 shows connection duration as a function of relative speed for different vehicle densities when observed nodes move in the same direction ${ }^{3}$. As expected, connection duration is directly dependent on the relative speed of the observed vehicles. Furthermore, connection duration usually benefits from higher vehicle densities, except in the scenario with relative speed zero, where increased vehicle density has a negative impact on connection duration because the nonobserved vehicles present obstacles that can slow down one of the observed vehicles; this makes the distance between the observed vehicles larger, thus increasing the probability of a connection outage (Fig. 5a). Fig. 6 shows that vehicles traveling in the opposite direction (i.e., moving at high relative speeds) benefit greatly from additional nodes that can relay the messages. Connection duration is up to three times longer with $10 \mathrm{veh} / \mathrm{km}$ compared to $0 \mathrm{veh} / \mathrm{km}$. The large difference in the connection duration between the same and opposite direction highway scenarios points to the fact that it is useful for a VANET application to distinguish between opposite and same direction traffic and to predict potential connection duration based on that knowledge.

In the urban environment, connection duration is highly dependent on the vehicle density, since the observed vehicles travel in two dimensions (as opposed to one dimension in the

\footnotetext{
${ }^{3}$ We note here that the line representing 0 veh $/ \mathrm{km}$ in Fig. 5 and Fig. 6 is simply a 'deterministic' value calculated as a quotient of the transmission radius and the relative speed of vehicles.
} 


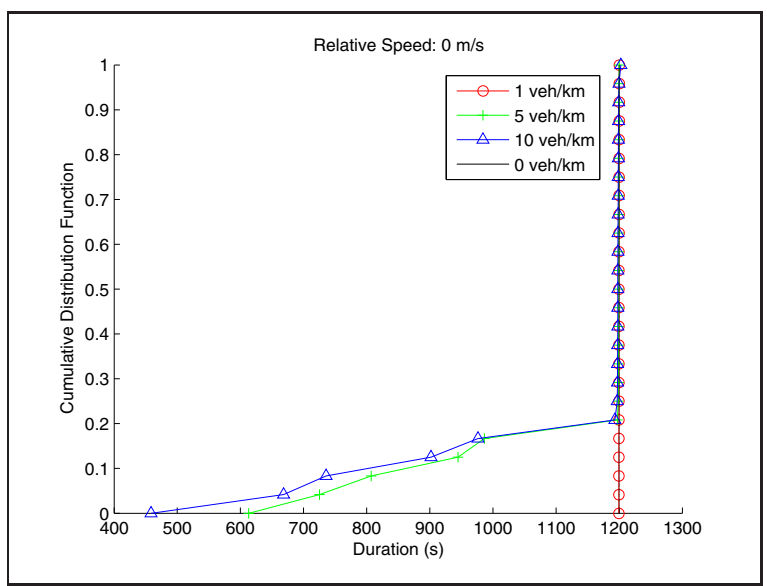

(a) Relative speed $=0 \mathrm{~m} / \mathrm{s}$

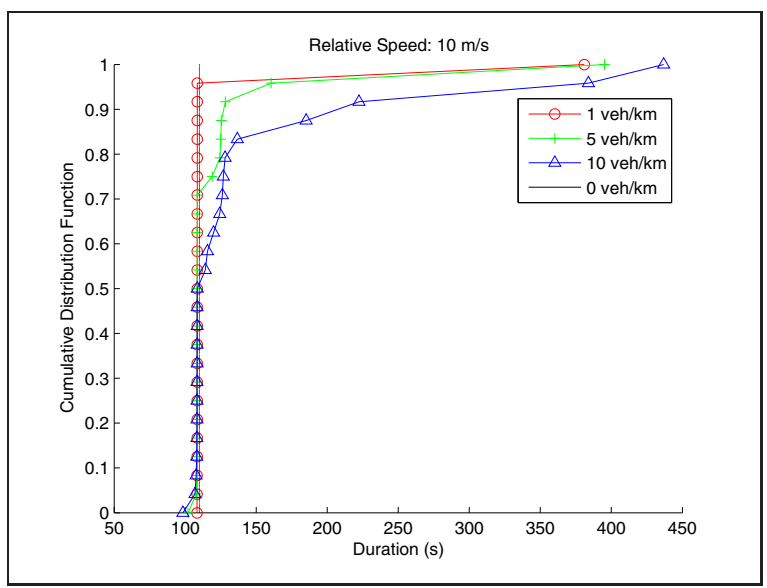

(c) Relative speed $=10 \mathrm{~m} / \mathrm{s}$

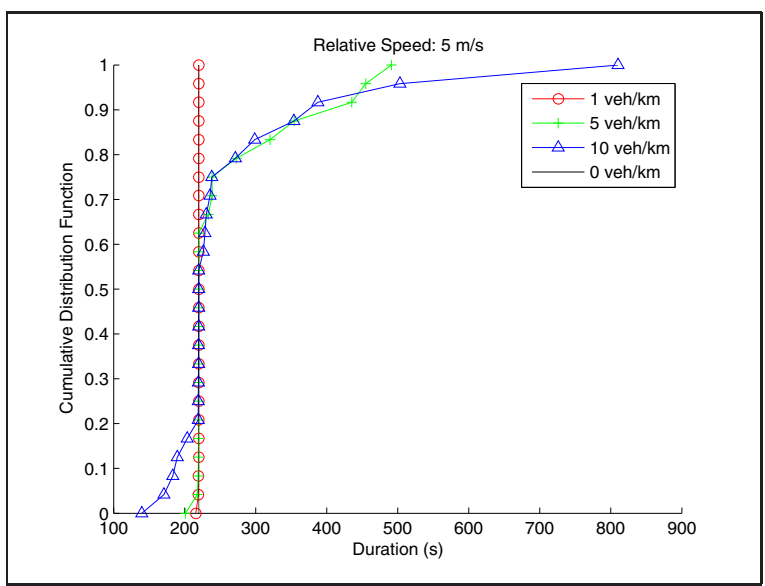

(b) Relative speed $=5 \mathrm{~m} / \mathrm{s}$

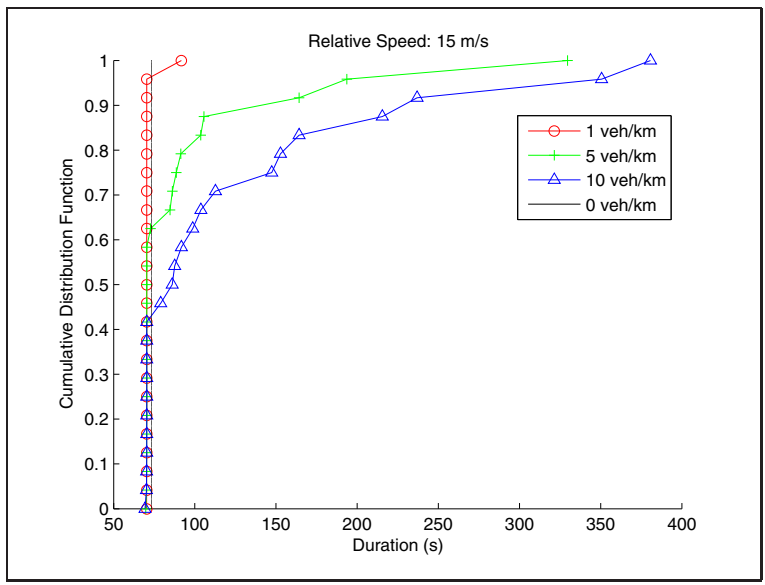

(d) Relative speed $=15 \mathrm{~m} / \mathrm{s}$

Fig. 5. Connection duration in the highway environment - same direction.

highway environment) and it cannot be assumed that they will travel in the same direction (or even meet on the road), thus the additional nodes are utilized to increase the connection duration. It is interesting to notice relatively low average connection durations for all four vehicle densities (see Fig. 7). Such short connection durations are result of the employed signal propagation model. The connection duration more than doubles if we choose the path loss exponent $\beta$ to be 2.4, which would correspond to an urban environment with smaller number of obstacles and less of an impact of the multipath propagation effects.

It is clear that in a complex urban environment, applications requiring connection durations of the order of minutes will not be able to function properly without the support of the infrastructure. However, according to the U.S. DOT infrastructure deployment plans [2], the first areas to be equipped with Roadside Units (RSUs) are the intersections of large U.S. cities, which will likely alleviate the connectivity problem.

With regards to the routing protocols, such a short connection duration in urban areas (of the order of seconds) implies that the route changes will occur at best at that frequency, thus making topology-based routing highly unlikely to perform well in infrastructureless VANET. We base this conclusion on the fact that the topology-based routing protocols in VANET would have to be able to change routes every several seconds in order to ensure connectivity: the overhead that would be created by such frequent route updates would be very high.

Similarly to connection duration, PDR is greatly affected by the vehicle density. Because the observed nodes can move over many different roads in different directions, the existence of nodes that can relay the message over a number of hops implies that a larger number of messages will be delivered. Fig. 8 clearly shows the relation of the vehicle density to PDR. Median values of PDR amounted to approximately 1\%, 3.5\%, $17 \%$, and $72 \%$ for the analyzed vehicle densities.

Fig. 9 shows the average delay compared to the distance between the observed nodes. It is obvious that the delay is highly dependent on the distance between the observed nodes. In general, the delay increases fairly linearly with distance, with a steeper slope for urban areas where the same physical distance between the observed nodes implies larger number of hops, and thus higher delay due to the increase in transmission delay. It is interesting to observe the maximum distances between nodes achieved for different 


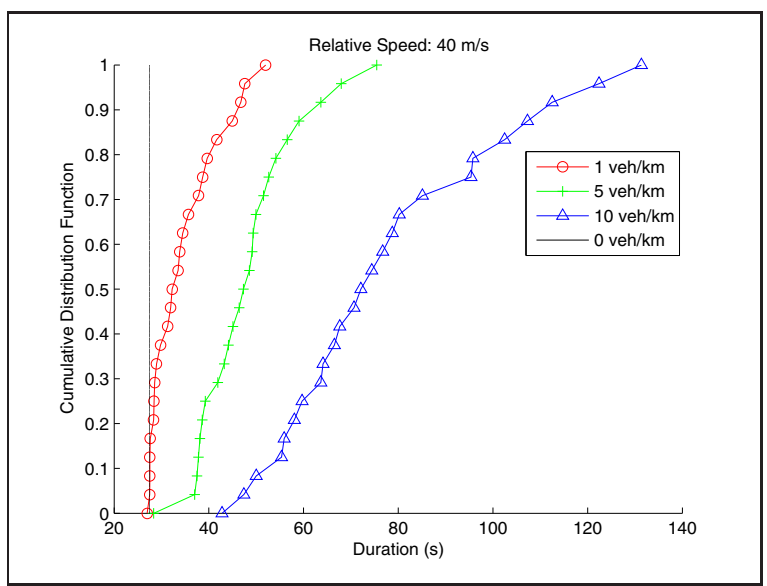

(a) Relative speed $=40 \mathrm{~m} / \mathrm{s}$

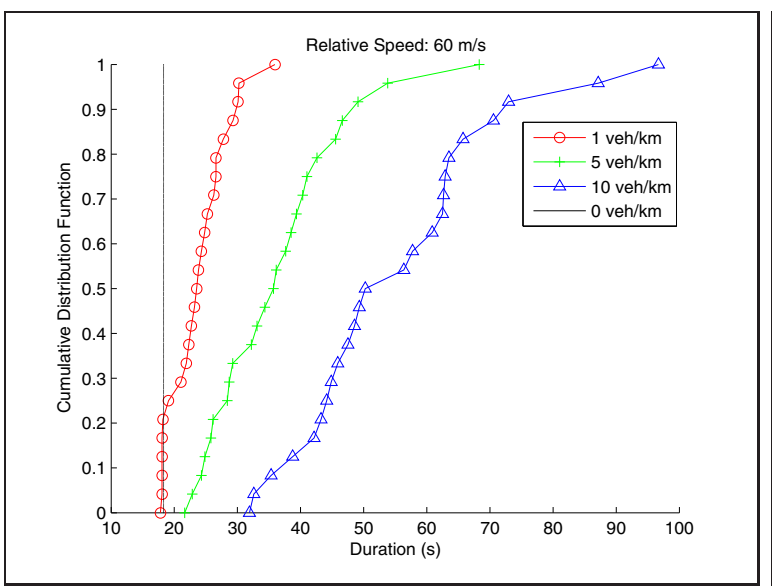

(c) Relative speed $=60 \mathrm{~m} / \mathrm{s}$

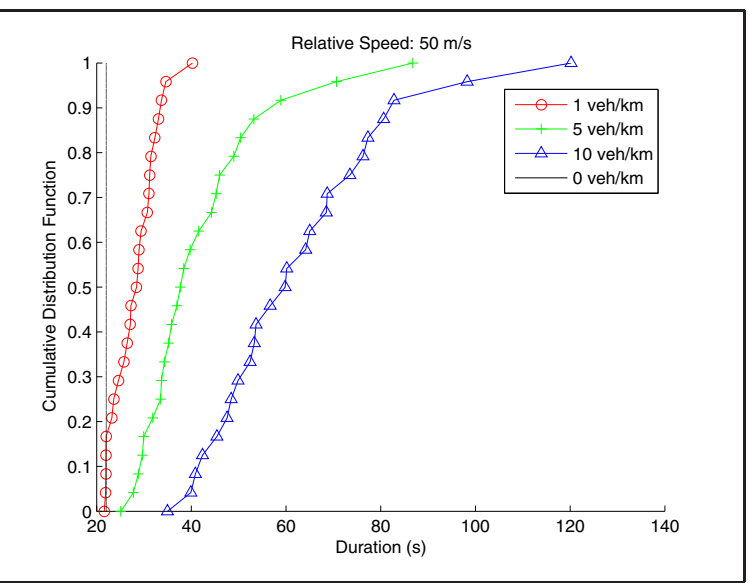

(b) Relative speed $=50 \mathrm{~m} / \mathrm{s}$

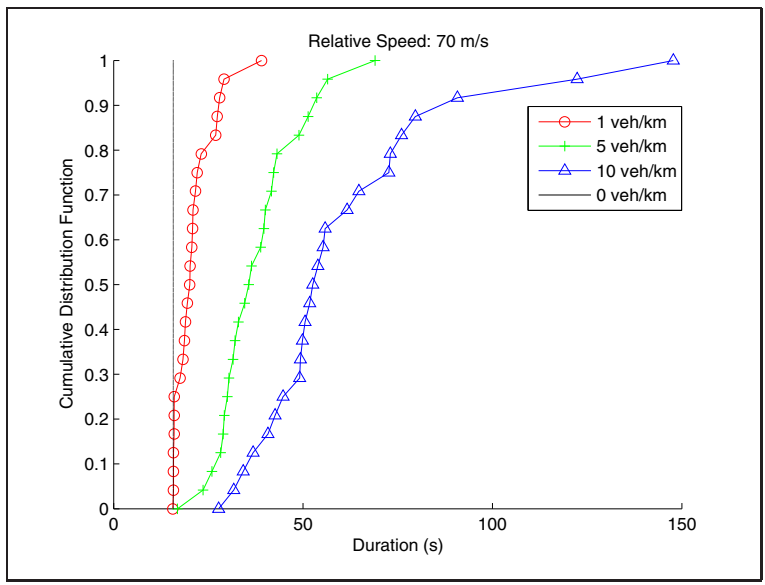

(d) Relative speed $=70 \mathrm{~m} / \mathrm{s}$

Fig. 6. Connection duration in the highway environment - opposite direction.

environments. Since the observed urban area was a square with the diagonal of $3.9 \mathrm{~km}$, the maximum distance between transmitting nodes cannot exceed that value. Also, given that the transmission range of radios in urban area was decreased compared to highway area, the maximum distance achievable is significantly shorter than in highway environment. Given that the connection duration was of the order of hundreds of seconds in the same direction highway scenarios, the maximum distance between the observed nodes appears to be quite small. The reason behind such a small maximum distance is that at low vehicle densities there are not enough intermediate nodes to support the communication over large distances, while at higher densities the traffic congestion on the roads makes the vehicles unable to travel at high relative speeds due to the employed mobility model. Furthermore, the maximum distance achieved in opposite direction highway scenarios confirms our hypothesis that applications in VANET are going to be bound to a certain region of interest; although at moments the observed vehicles were more than $10 \mathrm{~km}$ apart, even the employed routing protocol that accounts for all available paths was unable to relay the messages over distances larger than $4.5 \mathrm{~km}$.
Fig. 10 shows jitter distribution for urban and both same and opposite direction highway scenarios. Jitter is largely confined within $0-30 \mathrm{~ms}$ for highway and up to $50 \mathrm{~ms}$ in urban environment. The longer tail in the distribution of jitter in urban environment is again due to the decreased transmission range, which implies larger number of hops, thus producing larger variance.

The results presented in this section point out the ability of DSRC-enabled VANET to support one way end-to-end delay below $100 \mathrm{~ms}$, jitter largely below $40 \mathrm{~ms}$, and that given a high enough vehicle density in urban environment or a small enough locale of interest in highway environment, relatively high PDR may be expected. However, connection duration seems to be the key issue for enabling applications in VANET (especially real-time applications, which require a meaningful connection duration). In the case of high vehicle densities and low relative speed between vehicles on a highway, the connection duration can be several minutes. However, in cases of sparse network and high relative speeds (i.e., vehicles moving in the opposite direction on highway) as well as in urban areas, the connection duration is significantly shorter. Therefore, in order to achieve longer connection durations, 


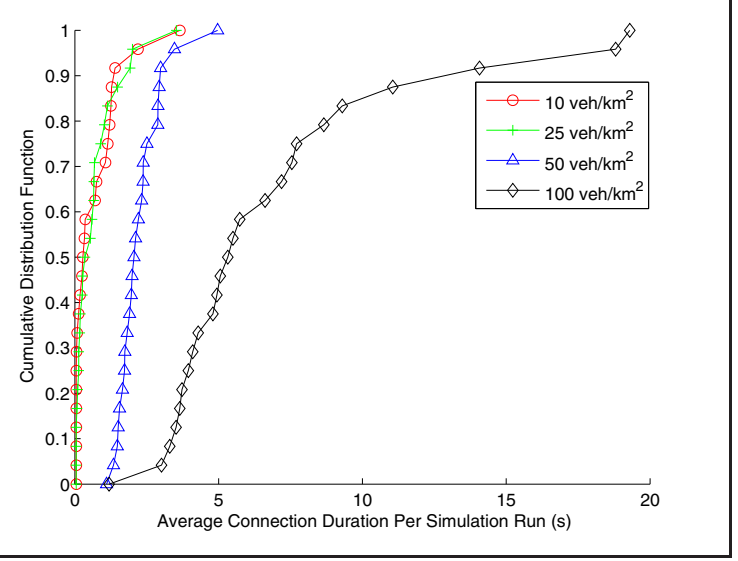

Fig. 7. Connection duration in the urban environment.

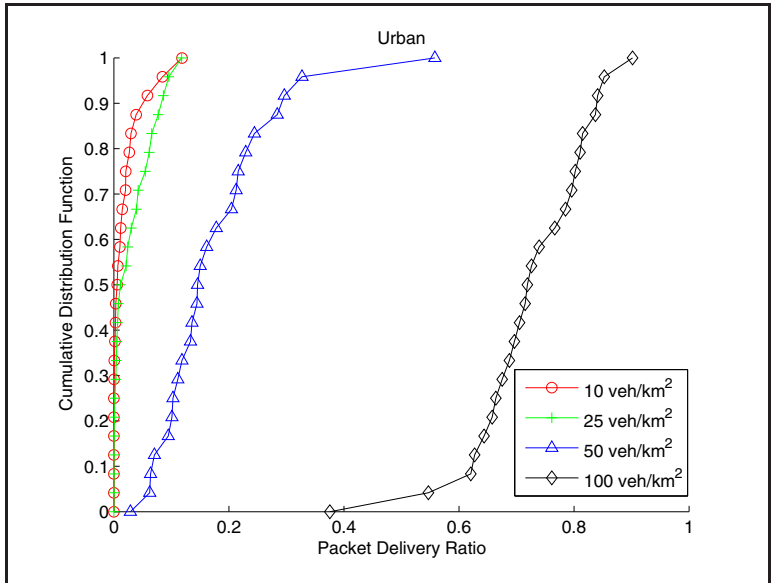

Fig. 8. Packet delivery ratio for different vehicle densities in the urban environment.

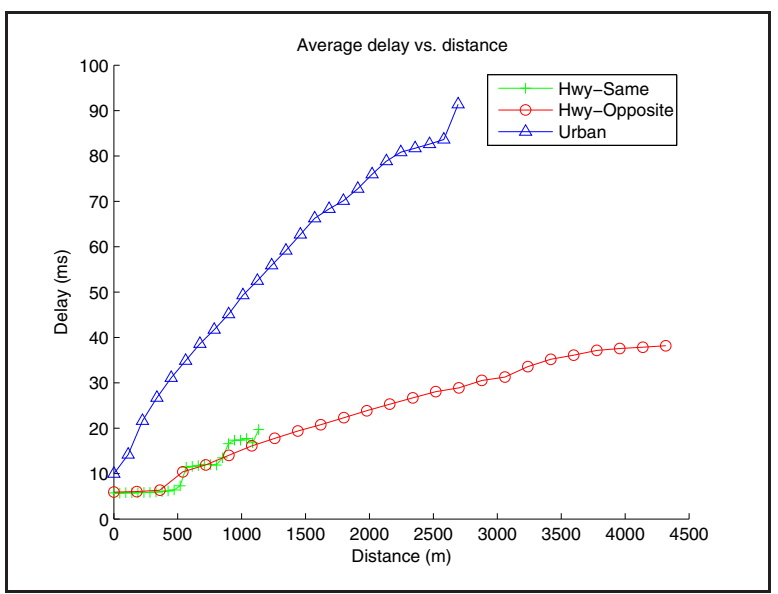

Fig. 9. Average end-to-end delay vs. distance between the observed nodes.

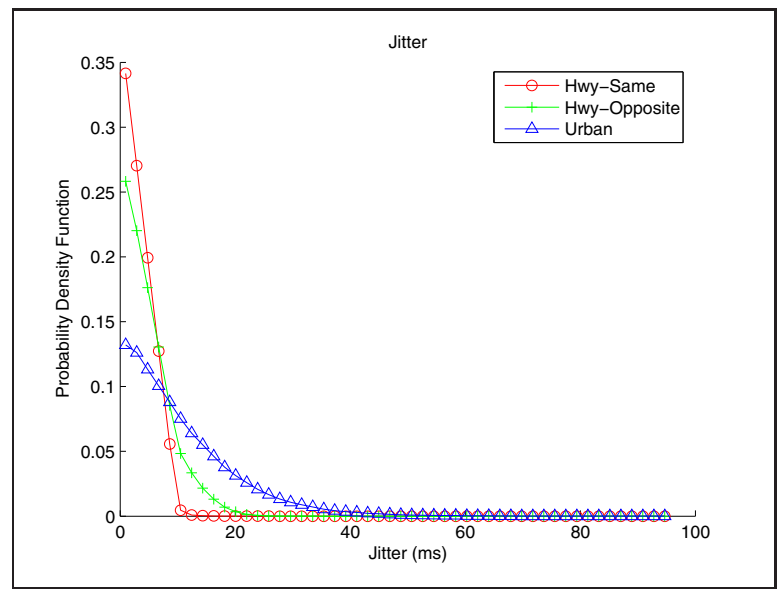

Fig. 10. The distribution of jitter in different environments.

infrastructure support will be necessary even over relatively small areas (the simulated urban area was $2.3 * 3.2 \mathrm{~km}^{2}$ ).

In a highway environment, message delivery over a geographical area larger than $4.5 \mathrm{~km}$ was not observed. This confirms our initial assumption that applications in VANET, especially real-time non-safety ones, will be bound to display locality of interest. In a sparse network this is true because of the lack of relaying nodes: store-carry-forward routing [32] could be used to increase the geographical range and PDR, but the delay and jitter would be exacerbated. In a dense network, however, relaying the message over a large number of hops could result in flooding the network, thus significantly affecting the network performance [33]. This is especially true for real-time applications, which create a continuous stream of messages.

Furthermore, based on the obtained results, we argue that topology-based routing is not a viable option in VANET since the dynamic VANET environment would require a very frequent route change, thus incurring additional delay and overhead by flooding the network with control packets in case of dense networks.

\section{CONClusions ANd Future Work}

In order to characterize the behavior of infrastructureless VANET, we presented comprehensive simulation results based on a realistic testbed setup. By conducting simulations in both highway and urban environments and by varying both the vehicles' speed and density, we were able to thoroughly analyze the important QoS metrics: connection duration, delay, PDR and jitter. We showed that values of delay and jitter in VANET can satisfy the requirements of most applications, while PDR and connection duration are both highly dependent on the vehicle density and the specific environment, and the connection duration is also closely related to the relative speed of vehicles. The results also confirmed our initial assumptions regarding the locality of interest for applications in VANET; communication over large area will not be possible without the use of infrastructure. 
Compared to the other ad hoc networks, due to its highly dynamic nature a VANET environment clearly presents great challenges in designing appropriate routing protocols. The results obtained are valuable because they define the upper performance bound for unicast routing over DSRC-enabled VANET in both urban and highway environments with typical vehicle speeds and traffic densities.

In the future, we plan to further elaborate on the redundant routes in urban and highway environment in order to determine the optimum rebroadcast probabilities for selective broadcast routing protocols in these environments. We are also going to analyze the QoS metrics in urban environment with respect to close proximity neighbors (i.e., one and two hop neighbors), in order to see whether restricting the geographical range of communication can assure increased PDR and connection duration even without infrastructure support. Furthermore, extending the employed routing scheme to support broadcast/multicast would provide a useful insight into the potential performance of many safety and non-safety applications in VANET that require such communication.

\section{REFERENCES}

[1] "IEEE Trial-Use Standard for Wireless Access in Vehicular Environments (WAVE) - Multi-channel Operation," IEEE Std 1609.4-2006, pp. c1-74, 2006.

[2] M. Cops, "Vehicle Infrastructure Integration (VII) - Bringing Vehicle Adhoc Network Technology on the road," The Third ACM International Workshop on Vehicular Ad Hoc Networks, Sep. 2006.

[3] Y. Cheng and T. Robertazzi, "Critical Connectivity Phenomena in Multihop Radio Models," IEEE Transactions on Communications, vol. 37, no. 7, pp. 770-777, Jul. 1989.

[4] S. Wang, "The effects of wireless transmission range on path lifetime in vehicle-formed mobile ad hoc networks on highways," IEEE International Conference on Communications, vol. 5, pp. 3177-3181 Vol. 5, 16-20 May 2005.

[5] S. Yousefi, E. Altman, R. El-Azouzi, and M. Fathy, "Analytical model for connectivity in vehicular ad hoc networks," submitted, 2007. [Online]. Available: http://wwwsop.inria.fr/maestro/personnel/Eitan.Altman/PAPERS/saleh-comnet.pdf

[6] F. Bai, N. Sadagopan, and A. Helmy, "IMPORTANT: a framework to systematically analyze the impact of mobility on performance of routing protocols for adhoc networks," INFOCOM 2003. Twenty-Second Annual Joint Conference of the IEEE Computer and Communications Societies. IEEE, vol. 2, pp. 825-835 vol.2, 30 March-3 April 2003.

[7] M. Artimy, W. Phillips, and W. Robertson, "Connectivity with static transmission range in vehicular ad hoc networks," Proceedings of the $3 \mathrm{rd}$ Annual Communication Networks and Services Research Conference, pp. 237-242, 16-18 May 2005.

[8] M. Rudack, M. Meincke, K. Jobmann, and M. Lott, "On traffic dynamical aspects of inter vehicle communications (IVC)," IEEE 58th Vehicular Technology Conference, VTC 2003-Fall. 2003, vol. 5, pp. 3368-3372, Oct. 2003.

[9] I. W. Ho, K. K. Leung, J. W. Polak, and R. Mangharam, "Node connectivity in vehicular ad hoc networks with structured mobility," 32nd IEEE Conference on Local Computer Networks, LCN 2007., pp. 635-642, 15-18 Oct. 2007.

[10] M. Mabiala, A. Busson, and V. Veque, "Inside vanet: Hybrid network dimensioning and routing protocol comparison," Vehicular Technology Conference, 2007. VTC2007-Spring. IEEE 65th, pp. 227-232, April 2007.

[11] M. Fiore and J. Härri, "The networking shape of vehicular mobility," in MobiHoc '08: Proceedings of the 9th ACM international symposium on Mobile ad hoc networking and computing. New York, NY, USA: ACM, 2008, pp. 261-272.
[12] J. Yin, T. ElBatt, G. Yeung, B. Ryu, S. Habermas, H. Krishnan, and T. Talty, "Performance evaluation of safety applications over DSRC vehicular ad hoc networks," in VANET '04: Proceedings of the 1st ACM international workshop on Vehicular ad hoc networks. New York, NY, USA: ACM, 2004, pp. 1-9.

[13] U. Lee, J.-S. Park, E. Amir, and M. Gerla, "Fleanet: A virtual market place on vehicular networks," 3rd Annual International Conference on Mobile and Ubiquitous Systems - Workshops, 2006., pp. 1-8, 17-21 July 2006.

[14] O. Tonguz and M. Boban, "Multiplayer games over VANET: a new application," submitted, 2008.

[15] M. Dikaiakos, A. Florides, T. Nadeem, and L. Iftode, "Location-aware services over vehicular ad-hoc networks using car-to-car communication," IEEE Journal on Selected Areas in Communications, vol. 25, no. 8, pp. $1590-1602$, Oct. 2007.

[16] V. Naumov, R. Baumann, and T. Gross, "An evaluation of inter-vehicle ad hoc networks based on realistic vehicular traces," in MobiHoc '06: Proceedings of the 7th ACM international symposium on Mobile ad hoc networking and computing. New York, NY, USA: ACM, 2006, pp. $108-119$.

[17] N. Wisitpongphan, F. Bai, P. Mudalige, V. Sadekar, and O. Tonguz, "Routing in Sparse Vehicular Ad Hoc Wireless Networks," IEEE Journal on Selected Areas in Communications, vol. 25, no. 8, pp. 1538-1556, Oct. 2007.

[18] W. Sun, H. Yamaguchi, K. Yukimasa, and S. Kusumoto, "GVGrid: A QoS routing protocol for vehicular ad hoc networks," 14th IEEE International Workshop on Quality of Service, 2006. IWQoS 2006., pp. 130-139, June 2006.

[19] F. Li and Y. Wang, "Routing in vehicular ad hoc networks: A survey," IEEE Vehicular Technology Magazine, vol. 2, no. 2, pp. 12-22, June 2007.

[20] B. Karp and H. T. Kung, "GPSR: greedy perimeter stateless routing for wireless networks," in MobiCom, 2000, pp. 243-254.

[21] R. Barr, Z. J. Haas, and R. van Renesse, "JiST: an efficient approach to simulation using virtual machines," Softw, Pract. Exper, vol. 35, no. 6, pp. 539-576, 2005.

[22] D. R. Choffnes and F. E. Bustamante, "An integrated mobility and traffic model for vehicular wireless networks," in VANET '05: Proceedings of the 2nd ACM international workshop on Vehicular ad hoc networks. New York, NY, USA: ACM, 2005, pp. 69-78.

[23] "U.S. census bureau TIGER system database." [Online]. Available: http://www.census.gov/geo/www/tiger

[24] R. W. Rothery, "Car following models," In Trac Flow Theory, 1992.

[25] "Standard Specification for Telecommunications and Information Exchange Between Roadside and Vehicle Systems - 5GHz Band Dedicated Short Range Communications (DSRC) Medium Access Control (MAC) and Physical Layer (PHY) Specifications," ASTM E2213-03, Sep. 2003.

[26] D. Kotz, C. Newport, R. S. Gray, J. Liu, Y. Yuan, and C. Elliott, "Experimental evaluation of wireless simulation assumptions," in MSWiM '04: Proceedings of the 7th ACM international symposium on Modeling, analysis and simulation of wireless and mobile systems. New York, NY, USA: ACM, 2004, pp. 78-82.

[27] "Vehicle Safety Communications Project, Final Report," U.S. Department of Transportation, NHTSA, Crash Avoidance Metrics Partnership, Tech. Rep. DOT HS 810 591, 2006.

[28] J. S. Davis and J. P. M. G. Linnartz, "Vehicle to vehicle RF propagation measurements," Twenty-Eighth Asilomar Conference on Signals, Systems, and Computers, vol. 1, Oct. 1994.

[29] J. Färber, "Traffic Modelling for Fast Action Network Games," Multimedia Tools and Applications, vol. 23, no. 1, pp. 31-46, 2004.

[30] “NGSIM freeway lane selection model," U.S. Department of Transportation, Federal Highway Administration, Tech. Rep. FHWA-HOP-06-103, Dec. 2004.

[31] "Identify Intelligent Vehicle Safety Applications Enabled by DSRC," U.S. Department of Transportation, NHTSA, Crash Avoidance Metrics Partnership, Tech. Rep. Task 3 Report, 2004.

[32] J. Wu, S. Yang, and F. Dai, "Logarithmic store-carry-forward routing in mobile ad hoc networks," IEEE Transactions on Parallel and Distributed Systems, vol. 18, no. 6, pp. 735-748, June 2007.

[33] N. Wisitpongphan, O. Tonguz, J. Parikh, P. Mudalige, F. Bai, and V. Sadekar, "Broadcast storm mitigation techniques in vehicular ad hoc networks," IEEE Wireless Communications, vol. 14, no. 6, pp. 84-94, December 2007. 